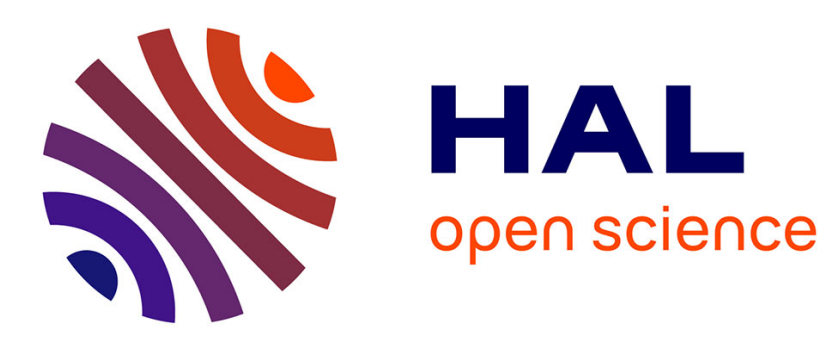

\title{
Continuous time opinion dynamics of agents with multi-leveled opinions and binary actions
}

Vineeth Varma, Irinel-Constantin Morarescu, Yezekael Hayel

\section{To cite this version:}

Vineeth Varma, Irinel-Constantin Morarescu, Yezekael Hayel. Continuous time opinion dynamics of agents with multi-leveled opinions and binary actions. IEEE Conference on Computer Communications, INFOCOM 2018, Apr 2018, Honolulu, United States. hal-01756831

\section{HAL Id: hal-01756831 \\ https://hal.science/hal-01756831}

Submitted on 3 Apr 2018

HAL is a multi-disciplinary open access archive for the deposit and dissemination of scientific research documents, whether they are published or not. The documents may come from teaching and research institutions in France or abroad, or from public or private research centers.
L'archive ouverte pluridisciplinaire HAL, est destinée au dépôt et à la diffusion de documents scientifiques de niveau recherche, publiés ou non, émanant des établissements d'enseignement et de recherche français ou étrangers, des laboratoires publics ou privés. 


\section{Continuous time opinion dynamics of agents with multi-leveled opinions and binary actions}

\author{
Vineeth S Varma and Irinel-Constantin Morărescu \\ CRAN UMR 7039, CNRS - Université de Lorraine, France \\ vineeth. satheeskumar-varma@univ-lorraine. fr
}

\author{
Yezekael Hayel \\ University of Avignon, Avignon, France
}

\begin{abstract}
This paper proposes and analyzes a stochastic multiagent opinion dynamics model. We are interested in a multileveled opinion of each agent which is randomly influenced by the binary actions of its neighbors. It is shown that, as far as the number of agents in the network is finite, the model asymptotically produces consensus. The consensus value corresponds to one of the absorbing states of the associated Markov system. However, when the number of agents is large, we emphasize that partial agreements are reached and these transient states are metastable, i.e., the expected persistence duration is arbitrarily large. These states are characterized using an $\mathrm{N}$-intertwined mean field approximation (NIMFA) for the Markov system. Numerical simulations validate the proposed analysis.
\end{abstract}

Index Terms-Opinion dynamics, Social computing and networks, Markov chains, agent based models

\section{INTRODUCTION}

Understanding opinion dynamics is a challenging problem that has received an increasing amount of attention over the past few decades. The main motivation of these studies is to provide reliable tools to fight against different addictions as well as propagation of undesired unsocial behaviors/beliefs. One of the major difficulties related to opinion dynamics is the development of models that can capture many features of a real social network [1]. Most of the existing models assume that individuals are influenced by the opinion of their neighbors [2], [3], [4], [5], [6], [7]. Nevertheless, it is very hard to estimate these opinions. In order to relax this constraint of measuring the opinions and to model more realistic behaviors, a mix of continuous opinion with discrete actions (CODA) was proposed in [8]. This model reflects the fact that even if we often face binary choices or actions which are visible by our neighbors, the opinions evolve in a continuous space of values which are not explicitly visible to the neighbors. A multi-agent system with a CODA model was proposed and analyzed in [9]. It was shown that this deterministic model leads to a variety of asymptotic behaviors including consensus.

Due to the complexity of the opinion dynamics, we believe that stochastic models are more suitable than deterministic ones. Indeed, we can propose a realistic deterministic update rule but many random events will still influence the interaction network and consequently the opinion dynamics. For this reason, we consider that it is important to reformulate the model from [9] in a stochastic framework, specifically, as an interactive Markov chain. Similar approaches for the Deffuant and Hegselmann-Krause models have been considered in the literature (see for instance [10], [11]). Although the asymptotic behavior of the model can be given by characterizing the absorbing states of the Markov chain, the convergence time can be arbitrarily large. Moreover, transient but persistent local agreements, called metastable equilibria, are very interesting because they describe the finite-time behavior of the network. Consequently, we consider in this paper an $\mathrm{N}$-intertwined mean field approximation (NIMFA) based approach in order to characterize the metastable equilibria of the Markov system. It is noteworthy that NIMFA was successfully used to analyze and validate some epidemiological models [12], [13].

In this work, we model the social network as a multi-agent system in which each agent represents an individual whose state is his opinion. This opinion can be understood as the preference of the agent towards performing a binary action, i.e. action can be 0 or 1 . These agents are interconnected through an interaction directed graph, whose edge weights represent the trust given by an agent to his neighbor. We propose continuous time opinion dynamics in which the opinions are discrete and belong to a given set that is fixed a priori. Each agent is influenced randomly by the actions of his neighboring agents and consequently influences its neighbors. Therefore the opinions of agents are an intrinsic variable that is hidden from the other agents, the only visible variable is the action. As an example, consider the opinion of users regarding two products Coca-cola and Pepsi. A user may prefer Coca-cola strongly, while some other users might be more indifferent. However, what the other users see (and is therefore influenced by) is only what the user buys, which is the action taken.

Our goal here is to analyze the behavior of the opinions in the network under the proposed stochastic dynamics. One of the main results states that the opinions always asymptotically reach a consensus defined by one of the extreme opinions. Nevertheless, for large networks, we emphasize that intermediate local agreements are reached and preserved for a long duration of time. The contributions of this paper can be summarized as follows. Firstly, we formulate and analyze a stochastic version of the CODA model proposed in [9]. Secondly, we characterize the local agreements which are persistent for a long duration by using the NIMFA for the original Markov system. Thirdly, we give a complete characterization of the system behavior under symmetric allto-all connection assumption. Finally, we provide conditions 
for the preservation of the main action inside one cluster as well as for the propagation of actions.

The rest of the paper is organized as follows. Section II introduces the main notation and concepts and provides a description of the model used throughout the paper. The analysis of the asymptotic behavior of opinions described by this stochastic model is provided in Section III. The presented results are valid for any connected networks with a finite number of agents. Moreover Section III contains the description of the NIMFA model and an method to compute its equilibria. In Section IV, we analyze the particular network in which each agent is connected to all the others. In this case, we show that only three equilibria exist and two of them are stable, and correspond to the absorbing states of the system. Section V presents the theoretical analysis of the system under generic interaction networks. It also emphasizes conditions for the preservation of the main action (corresponding to a metastable state) in some clusters as well as conditions for the action propagation. The results of our work are numerically illustrated in Section VI. The paper ends with some concluding remarks and perspectives for further developments.

Preliminaries: We use E for the expectation of a random variable, $\mathbf{1}_{A}(x)$ the indicator function which takes the value 1 when $x \in A$ and 0 otherwise, $\mathbb{R}_{+}$the set of non-negative reals and $\mathbb{N}=\{1,2, \ldots\}$ the set of natural numbers..

\section{MODEL}

Throughout the paper we consider $N \in \mathbb{N}$ an even number of possible opinion levels, and the set of agents $\mathcal{K}=\{1,2, \ldots, K\}$ with $K \in \mathbb{N}$. Each agent $i$ is characterized at time $t \in \mathbb{R}_{+}$by its opinion represented as a scalar $X_{i}(t) \in \Theta$ where $\Theta=\left\{\theta_{1}, \theta_{2}, \ldots, \theta_{N}\right\}$ is the discrete set of possible opinions, such that $\theta_{n} \in(0,1) \backslash\{0.5\}$ and $\theta_{n}<\theta_{n+1}$ for all $n \in\{1,2, \ldots, N\}$. Moreover $\Theta$ is constructed such that $\theta_{N / 2}<0.5$ and $\theta_{N / 2+1}>0.5$. In the following let us introduce some graph notions allowing us to define the interaction structure in the social network under consideration.

Definition 1 (Directed graph): A weighted directed graph $G$ is a couple $(\mathcal{K}, A)$ with $\mathcal{K}$ being a finite set denoting the vertices, and $A$ being a $K \times K$ matrix, with elements $a_{i j}$ denoting the trust given by $i$ on $j$. We say that agent $j$ is a neighbor of agent $i$ if $a_{i j}>0$.

We denote by $\tau_{i}$ the total trust in the network for agent $i$ as $\tau_{i}=\sum_{j=1}^{K} a_{i j}$. Agent $i$ is said to be connected with agent $j$ if $G$ contains a directed path from $i$ to $j$, i.e. if there exists at least one sequence $\left(i=i_{1}, i_{2}, \ldots, i_{p+1}=j\right)$ such that $a_{i_{k}, i_{k+1}}>0, \forall k \in\{1,2, \ldots, p\}$.

Definition 2 (Strongly connected): The graph $G$ is strongly connected if any two distinct agents $i, j \in \mathcal{K}$ are connected. In the sequel we suppose the following holds true.

Assumption 1: The graph $(\mathcal{K}, \mathcal{E})$ modeling the interaction in the network is strongly connected.

\begin{tabular}{|c|c|}
\hline Symbol & Meaning \\
\hline$\Theta$ & Set of opinion values, $|\Theta|=N$, with $N$ even. \\
\hline $\mathcal{K}$ & Set of agents, $|\mathcal{K}|=K$ \\
\hline$\beta_{n}$ & Willingness to shift opinion, with current opinion $\theta_{n} \in \Theta$ \\
\hline$X_{i}(t)$ & Opinion of agent $i$ at time $t, X_{i}(t) \in \Theta$ \\
\hline$Q_{i}(t)$ & $\begin{array}{l}\text { Action of agent } i \text { at time } t \\
Q_{i}(t)=\left\lfloor X_{i}(t)\right\rceil \in\{0,1\}\end{array}$ \\
\hline$a_{i j}$ & Trust of agent $i$ in agent $j$ \\
\hline$\tau_{i}$ & $\begin{array}{l}\text { Trust of agent } i \text { in the network } \\
\qquad \tau_{i}=\sum_{j \in \mathcal{K}} a_{i j}\end{array}$ \\
\hline$v_{i, n}(t)$ & Probability that $X_{i}(t)=\theta_{n}$ \\
\hline$R_{i}(t)$ & $\begin{array}{l}\text { Influence on } i \text { to shift opinion to } 1 \\
R_{i}(t)=\sum_{j \in \mathcal{K}} a_{i j} Q_{j}(t)\end{array}$ \\
\hline$L_{i}(t)$ & $\begin{array}{l}\text { Influence on } i \text { to shift opinion to } 0 \\
L_{i}(t)=\sum_{j \in \mathcal{K}} a_{i j}\left(1-Q_{j}(t)\right)\end{array}$ \\
\hline$r_{i}(t)$ & $\begin{array}{l}\text { Expected influence on } i \text { to shift opinion to } 1 \\
r_{i}(t)=\sum_{j \in \mathcal{K}} a_{i j} \sum_{n=N / 2+1}^{N} v_{j, n}(t)\end{array}$ \\
\hline$l_{i}(t)$ & $\begin{array}{l}\text { Expected influence on } i \text { to shift opinion to } 0 \\
\quad l_{i}(t)=\sum_{j \in \mathcal{K}} a_{i j} \sum_{n=1}^{N / 2} v_{j, n}(t)\end{array}$ \\
\hline$\nu_{n}(t)$ & $\begin{array}{l}\text { Expected fraction of population } \\
\text { with opinion } \theta_{n}, \nu_{n}=\sum_{i \in \mathcal{K}} \frac{v_{i, n}(t)}{K}\end{array}$ \\
\hline $\begin{array}{l}\nu_{+}(t) \\
\nu_{-}(t)\end{array}$ & $\begin{array}{c}\text { Expected fraction of population } \\
\text { with action } 1, \nu_{+}(t)=\sum_{i \in \mathcal{K}} \sum_{n=N / 2+1}^{N} \frac{v_{i, n}(t)}{K} \\
\text { or action } 0, \nu_{-}=\sum_{i \in \mathcal{K}} \sum_{n=1}^{N / 2} \frac{v_{i, n}(t)}{K}\end{array}$ \\
\hline$\nu_{n}^{C}(t)$ & $\begin{array}{l}\text { Expected fraction of population in } C \subseteq \mathcal{K} \\
\text { with opinion } \theta_{n}, \nu_{n}^{C}(t)=\sum_{i \in C} \frac{v_{i, n}(t)}{|C|}\end{array}$ \\
\hline $\begin{array}{l}\nu_{+}^{C}(t) \\
\nu_{-}^{C}(t)\end{array}$ & $\begin{array}{l}\text { Expected fraction of population in } C \subseteq \mathcal{K} \\
\text { with action } 1, \nu_{+}^{C}=\sum_{i \in C} \sum_{n=N / 2+1}^{N} \frac{v_{i, n}(t)}{|C|} \\
\text { or action } 0, \nu_{-}^{C}=\sum_{i \in C} \sum_{n=1}^{N / 2} \frac{v_{i, n}(t)}{|C|}\end{array}$ \\
\hline
\end{tabular}

TABLE I: Notations used

The action $Q_{i}(t)$ taken by agent $i$ at time $t$ is defined by the opinion $X_{i}(t)$ through the following relation

$$
Q_{i}(t)=\left\lfloor X_{i}(t)\right\rceil
$$

where $\lfloor$.$\rceil is the nearest integer function. This means that$ if an agent has an opinion more than 0.5 , it will take the action 1 and 0 otherwise. This kind of opinion quantization is suitable for many practical applications. For example, an agent may support the left or right political party, with various opinion levels (opinions close to 0 or 1 represents a stronger preference), however, in the election, the agent's action is to vote with exactly two choices (left or right). Similarly, an agent might have to choose between two cars or other types of merchandise like cola as mentioned in the introduction. Although its preference for one product is not of the type 0 or 1 , its action will be, since it cannot buy fractions of cars, but one of them. For ease of exposition, we provide Table I, a list of notations and their meanings.

\section{A. Opinion dynamics}

In this work, we look at the evolution of opinions of the agents based on their mutual influence. We also account for the inertia of opinion, i.e., when the opinion of the agent is closer to 0.5 , he is more likely to shift as he is less decisive, whereas someone with a strong opinion (close to 1 or 0 ) is less likely to shift his opinion as he is more convinced by his opinion. The opinion of agent $j$ may shift 
towards the actions of its neighbors with a rate $\beta_{n}$ while $X_{j}(t)=\theta_{n}$. If no action is naturally preferred by the opinion dynamics, then we construct $\theta_{n}=\theta_{N+1-n}$ and assume that $\beta_{n}=\beta_{N+1-n}$ for all $n \in\{1,2, \ldots, N\}$. At each time $t \in \mathbb{R}_{+}$ we denote the vector collecting all the opinions in the network by $X(t)=\left(X_{1}(t), \ldots, X_{K}(t)\right)$. Notice that the evolution of $X(t)$ is described by a continuous time Markov process with $N^{K}$ states and its analysis is complicated even for small number opinion levels and relatively small number of agents. The stochastic transition rate of agent $i$ shifting its opinion to the right, i.e. to have opinion $\theta_{n+1}$ when at opinion $\theta_{n}$, with $n \in\{1,2, \ldots, N-1\}$, is given by

$$
\beta_{n} \sum_{j=1}^{N} a_{i j} \mathbf{1}_{(0.5,1]}\left(X_{j}(t)\right)=\beta_{n} \sum_{j=1}^{K} a_{i j} Q_{j}(t)=\beta_{n} R_{i}(t) .
$$

Similarly, the transition rate to the left, i.e. to shift from $\theta_{n}$ to $\theta_{n-1}$ is given by

$\beta_{n} \sum_{j=1}^{N} a_{i j} \mathbf{1}_{[0,0.5)}\left(X_{j}(t)\right)=\beta_{n} \sum_{j=1}^{N} a_{i j}\left(1-Q_{j}(t)\right)=\beta_{n} L_{i}(t)$.

for $n \in\{2, \ldots, N\}$. Therefore, we can write the infinitesimal generator $M_{i, t}$ (a tri-diagonal matrix of size $N \times N$ ) for an agent $i$ as:

$$
M_{i, t}=\left(\begin{array}{cccc}
-\beta_{1} R_{i}(t) & \beta_{1} R_{i}(t) & 0 & \ldots \\
\beta_{2} L_{i}(t) & -\beta_{2} \tau_{i} & \beta_{2} R_{i}(t) & \ldots \\
\vdots & & &
\end{array}\right)
$$

with elements corresponding the $n$-th rown and $m$-th column given by $M_{i, t}(m, n)$ and

$$
\begin{gathered}
\forall n \in\{1, \ldots, N-1\}, \quad M_{i, t}(n, n+1)=\beta_{n} R_{i}(t), \\
\forall n \in\{2, \ldots, N\}, \quad M_{i, t}(n, n-1)=\beta_{n} L_{i}(t), \\
\forall|m-n|>1, \quad M_{i, t}(m, n)=0
\end{gathered}
$$

and

$$
M_{i, t}(n, n)=\left\{\begin{array}{lll}
-\beta_{1} R_{i}(t) & \text { for } & n=1 \\
-\beta_{n} \tau_{i} & \text { for } & n \in\{2, \ldots, N-1\}, \\
-\beta_{N} L_{i}(t) & \text { for } & n=N .
\end{array}\right.
$$

Let $v_{i, n}(t):=\mathrm{E}\left[\mathbf{1}_{\left\{\theta_{n}\right\}}\left(X_{i}(t)\right)\right]=\operatorname{Pr}\left(X_{i}(t)=\theta_{n}\right)$ be the probability for an opinion level $\theta_{n}$ for user $i$ at time $t$. Then, in order to propose an analysis of the stochastic process introduced above, we may consider the mean-field approximation by replacing the transitions by their expectations. Then, the expected transition rate from state $n$ to state $n+1$ for $K \rightarrow \infty$, is given by:

$$
\beta_{n} \sum_{j=1}^{K} a_{i j} \mathrm{E}\left[\mathbf{1}_{(0.5,1]}\left(X_{j}(t)\right)\right]=\beta_{n} \sum_{j=1}^{K} a_{i j} \sum_{n=N / 2+1}^{N} v_{j, n}(t) .
$$

We have similar expression for transition between state $n$ and $n-1$.

\section{STEAdy STATE ANALYSiS}

Define by $\bar{\theta}_{-}=\left(\theta_{1}, \ldots, \theta_{1}\right)$ and $\bar{\theta}_{+}=\left(\theta_{N}, \ldots, \theta_{N}\right)$ the states where all the agents in the network have an identical opinion, which correspond to the two extreme opinions.

Proposition 1: Under Assumption 1, the continuous time Markov process $X(t)$, with (1) as the infinitesimal generators corresponding to each agent, has exactly two absorbing states $X(t)=\bar{\theta}_{+}$and $X(t)=\bar{\theta}_{-}$.

Proof: We can verify that $\bar{\theta}_{+}$and $\bar{\theta}_{-}$are absorbing states by evaluating the transition rates using (1). If $X(t)=\bar{\theta}_{-}$, then $X_{i}(t)=\theta_{1}$ for all $i$, and the transition rate is

$$
(1,0, \ldots, 0) M_{i, t}=\left(-\beta_{1} R_{i}(t), \beta_{1} R_{i}(t), 0, \ldots, 0\right) .
$$

but as $X_{i}=\theta_{1}$ for all $i, Q_{i}(t)=0$ for all $i$ and so we have $R_{i}(t)=0$ for all $i$. Therefore the transition rate from this state is 0 . We can similarly show that $\bar{\theta}_{+}$is also an absorbing state.

Next, we show that no other state can be an absorbing state. Consider any state with at least one agent $i$ such that $X_{i}(t)=$ $\theta_{n}$ with $1<n<N$. The transition rate from such a state is never 0 , which can is easy to see, as we have $M_{i, t}(n, n)=$ $\beta_{n} \tau_{i}$.

This implies that as long as such an agent exists, the global state is not an absorbing state. The only states which are not $\bar{\theta}_{+}, \bar{\theta}_{-}$or such a state are those satisfying the following property. Consider $X_{i}(t)=\theta_{1}$ for all $i \in S$ and $X_{i}(t)=\theta_{N}$ for all $i \in \mathcal{K} \backslash S$ with $S \subset \mathcal{K}$ and $1<|S|<K$. As the graph is strongly connected, there is at least one agent $k$ in $S$, which is directly connected to some agent $l$ in $\mathcal{K} \backslash S$, i.e. $a_{k, l}>0$. The transition rate of this $k$ is given by

$$
\left(-\beta_{1} R_{k}(t), \beta_{1} R_{k}(t), 0, \ldots, 0\right)
$$

As $a_{k, l}>0$ and $Q_{l}(t)=1$ as all agents outside $S$ has opinion $\theta_{N}$, we have $R_{k}(t)>0$. Therefore such a state is also never an absorbing state, which concludes our proof that $\bar{\theta}_{+}$and $\bar{\theta}_{-}$are the two absorbing states and no other state is an absorbing state.

Considering the NIMFA approximation, we get that the dynamics of the opinion for an agent $i$ are given by:

$$
\begin{aligned}
\dot{v}_{i, 1}= & -\beta_{1} r_{i} v_{i, 1}+\beta_{2} l_{i} v_{i, 2} \\
\dot{v}_{i, n}= & -\beta_{n} r_{i} v_{i, n}+\beta_{n+1} l_{i} v_{i, n+1} \\
& -\beta_{n} l_{i} v_{i, n}+\beta_{n-1} r_{i} v_{i, n-1} \\
\dot{v}_{i, N}= & -\beta_{N} l_{i} v_{i, N}+\beta_{N-1} r_{i} v_{i, N-1}
\end{aligned}
$$

for all $i \in \mathcal{K}$ and $1<n<N$ where

$$
\begin{aligned}
l_{i} & =\sum_{j \in \mathcal{K}} a_{i j} E\left[1-Q_{j}\right] \\
& =\sum_{j \in \mathcal{K}} \sum_{n=1}^{N / 2} a_{i j} v_{j, n} \\
r_{i} & =\sum_{j \in \mathcal{K}} a_{i j} E\left[Q_{j}\right] \\
& =\sum_{j \in \mathcal{K}} \sum_{n=N / 2+1}^{N} a_{i j} v_{j, n} .
\end{aligned}
$$

and $\sum_{n} v_{i, n}=1$. 
We can easily verify that $X_{i}=\theta_{1}$, i.e. $v_{i, 1}=1$ for all $i$ is an equilibrium for the above set of equations. When $v_{i, 1}=1$ for all $i, v_{i, n}=0$ for all $n \geq 2$ and as a result, $l_{i}=\tau_{i}$ and $r_{i}=0$ for all $i$ which gives $\dot{v}_{i, n}=0$ for all $i, n$. Apart from the extreme solutions $\bar{\theta}_{+}$and $\bar{\theta}_{-}$, the non-linearity of system (3) could give rise to the existence of interior rest points which are locally stable. Such rest points are referred to as metastable states in Physics. Metastability of Markov processes is precisely defined in [14], where the exit times from these metastable states are shown to approach infinity.

For a given $r_{i}=E\left[R_{i}(t)\right]$, the equilibrium state $v_{i, n}^{*}$ must satisfy the following conditions

$$
\begin{aligned}
0= & -\beta_{1} \frac{r_{i}}{\tau_{i}} v_{i, 1}^{*}+\beta_{2}\left(\frac{\tau_{i}-r_{i}}{\tau_{i}}\right) v_{i, 2}^{*} \\
0= & -\beta_{n} v_{i, n}^{*}+\beta_{n+1}\left(\frac{\tau_{i}-r_{i}}{\tau_{i}}\right) v_{i, n+1} \\
& +\beta_{n-1} \frac{r_{i}}{\tau_{i}} v_{i, n-1}^{*} \\
0= & -\beta_{N}\left(\frac{\tau_{i}-r_{i}}{\tau_{i}}\right) v_{i, N}^{*}+\beta_{N-1} \frac{r_{i}}{\tau_{i}} v_{i, N-1}^{*}
\end{aligned}
$$

We can write any $v_{i, n}^{*}$ based on $v_{i, 1}^{*}$, by simplification as

$$
v_{i, n}^{*}=\frac{\beta_{1}}{\beta_{n}}\left(\frac{r_{i}}{\tau_{i}-r_{i}}\right)^{n-1} v_{i, 1}^{*} .
$$

As the sum of $v_{i, n}$ over $n$ must be 1 , we can solve for $v_{i, 1}^{*}$ as

$$
v_{i, 1}^{*}=\frac{1}{\sum_{n=1}^{N} \frac{\beta_{1}}{\beta_{n}}\left(\frac{r_{i}}{\tau_{i}-r_{i}}\right)^{n-1}} .
$$

We then can use this relationship to construct a fixed-point algorithm that computes a rest-point of the global opinion dynamics for all users.

Algorithm outline: The algorithm involves initializing $v$ to a random value. Then, this $v$ can be used to compute the corresponding $r_{i}$ and $l_{i}$ with (4), which is then used to compute the associated $v$ with (6). Repeating this recursively results in a fixed point of the NIMFA which is a potential metastable equilibrium. Other potential equilibria can be found by initializing with another random $v$.

Additionally, we can obtain some nice properties on the relation between $r_{i}$ and $v_{i, n}$ by studying the following function.

Lemma 1: Consider the function $f:[0,1] \rightarrow[0,1]$ defined as

$$
f(x):=\frac{\sum_{n=N / 2+1}^{N} \frac{\beta_{1}}{\beta_{n}}\left(\frac{x}{1-x}\right)^{n-1}}{\sum_{n=1}^{N} \frac{\beta_{1}}{\beta_{n}}\left(\frac{x}{1-x}\right)^{n-1}}
$$

for all $x \in[0,1)$ and with $f(1)=1$. We have that $f(x)$ is a monotonically increasing continuous function and takes the values $f(0)=0, f(0.5)=0.5$ and $\lim _{x \rightarrow 1} f(x)=1$.

Proof:

We can easily verify that

$$
f(0)=\frac{0}{1+0+\ldots}=0
$$

As $\beta_{n}$ is assumed to be symmetric around $N / 2$, i.e. $\beta_{1}=\beta_{N}$ etc., we have

$$
f(0.5)=\frac{\sum_{N / 2+1}^{N} \beta_{n}}{\sum_{1}^{N} \beta_{n}}=0.5
$$

In order to simplify differentiations, we use some additional variables, $\xi=\frac{x}{1-x}$,

$$
p=\beta_{1}+\beta_{2} \xi+\ldots \beta_{N / 2} \xi^{N / 2-1}
$$

and

$$
q=\beta_{1} \xi^{N}+\beta_{2} \xi^{N-1}+\ldots \beta_{N / 2} \xi^{N / 2}
$$

which gives $f(x)=\frac{q}{p+q}$. As $q=1+\ldots, \frac{q}{p+q}$ is continuous at all points except when $\xi$ is a pole, i.e., when $x=1$. Therefore if we show that $f(x)$ is continuous at $x=1, f(x)$ is continious is $[0,1]$. For this, we use L'Hôpital's rule to calculate $\lim _{x \rightarrow 1} f(x)$, which is

$$
\lim _{\xi \rightarrow \infty} \frac{\beta_{1} \xi^{N}+\beta_{2} \xi^{N-1}+\ldots \beta_{N / 2} \xi^{N / 2}}{\beta_{1}+\beta_{2} \xi+\ldots \beta_{N} \xi^{N}}
$$

Applying L'Hôpital's rule recursively $N$ times, we get this to be 1 . Therefore, we have shown that our function $f(x)$ is continuous in $[0,1]$. Next, we show that it is monotonic. We have

$$
\begin{aligned}
(f)^{\prime} & =\frac{q^{\prime}(p+q)-\left(p^{\prime}+q^{\prime}\right) q}{(p+q)^{2}} \\
& =\left(\frac{q}{p}\right)^{\prime} \frac{p^{2}}{(p+q)^{2}}
\end{aligned}
$$

where $(\cdot)^{\prime}=\frac{d(\cdot)}{d \xi}$. We see that $\frac{d \xi}{d x} \geq 0$. Therefore, if $\left(\frac{q}{p}\right)^{\prime} \geq$ 0 , then we have the monotonicity and increasing property. This can be verified by simplifying it as

$$
\left(\frac{q}{p}\right)^{\prime}=\sum_{n=N / 2+1}^{N}\left(\frac{\beta_{n} \xi^{n-1}}{p}\right)^{\prime}
$$

Each of these terms in the sum are positive and so we have shown that the first derivative of $f(x)$ w.r.t $x$ is positive.

We can use $f\left(\frac{r_{i}}{\tau_{i}-r_{i}}\right)$ to calculate the probability that an agent $i$ will take the action 1 , i.e., $\sum_{n=N / 2+1}^{N} v_{i, n}^{*}=f\left(\frac{r_{i}}{\tau_{i}-r_{i}}\right)$ from (7) and (6).

\section{COMPLETE GRAPH WITH IDENTICAL CONNECTIONS}

Let us first consider a simple graph structure in which each agent is identically influenced by all the others agents i.e., with $a_{i, j}=1$ for all $i, j \in \mathcal{K}$ and $i \neq j$, and $a_{i, i}=0$. We use $\nu_{n}$ to denote the expected fraction of the population of agents with opinion $\theta_{n}$. This is simply

$$
\nu_{n}=\frac{E\left[\sum_{i \in \mathcal{K}} \mathbf{1}\left(X_{i}=\theta_{n}\right)\right]}{K}=\frac{\sum_{i \in \mathcal{K}} v_{i, n}}{K} .
$$

Also recall that we introduced the following notation $\nu_{-}:=$ $\left(\sum_{n=1}^{N / 2} \nu_{n}\right)$ and $\nu_{+}=\left(\sum_{n=N / 2+1}^{N} \nu_{n}\right)$. Under this specific graph structure, we have 


$$
\begin{aligned}
\lim _{K \rightarrow \infty} \frac{r_{i}}{\tau_{i}} & =\frac{1}{\tau_{i}} \sum_{j \in \mathcal{K}} a_{i j} \sum_{n=N / 2+1}^{N} v_{j, n} \\
& =\nu_{+}-\frac{\sum_{n=N / 2+1}^{N} v_{i, n}}{K}=\nu_{+}
\end{aligned}
$$

for any $i \in \mathcal{K}$. Therefore, using (3) and the fact that $\dot{\nu}_{n}=$ $\sum_{i \in \mathcal{K}} \frac{\dot{v}_{i, n}}{K}$ we can get the dynamics of $\dot{\nu}_{n}, \forall n \in \mathcal{K}$. Moreover, following (12), for large values of $K$ we can approximate this dynamics as:

$$
\begin{aligned}
\frac{\dot{\nu}_{1}}{K-1}= & -\beta_{1} \nu_{1} \nu_{+}+\beta_{2} \nu_{2} \nu_{-}, \\
\frac{\dot{\nu}_{n}}{K-1}= & -\beta_{n} \nu_{n}+\beta_{n+1} \nu_{n+1} \nu_{-} \\
& +\beta_{n-1} \nu_{n-1} \nu_{+}, \quad \forall n \in\{2,3, \ldots, N-1\}, \\
\frac{\dot{\nu}_{N}=}{K-1}= & -\beta_{N} \nu_{N} \nu_{-}+\beta_{N-1} \nu_{N-1} \nu_{+} .
\end{aligned}
$$

where $\nu_{+}=\sum_{n=N / 2+1}^{N} \nu_{n}$ and $\nu_{-}=\sum_{n=1}^{N / 2} \nu_{n}$.

Theorem 1: When $N \geq 4, \beta_{n}>0$ and $\beta_{n}=\beta_{N-n+1}$ for all $n \in\{1,2, \ldots, N\}$, the dynamics described in (13) has exactly three equilibrium points at $(1,0, \ldots),(0, \ldots, 0,1)$ and at a point with $\nu_{+}=\nu_{-}=0.5$. The first two equilibrium points correspond to the absorbing states and are stable, while the third fixed point is an unstable equilibrium (not metastable).

Proof: Let us notice that:

- if $\nu_{1}=1$ and $\nu_{n}=0$ for all $n>1$ one gets $\nu_{+}=0$;

- if $\nu_{N}=1$ with $\nu_{n}=0$ for all $n<N$ one gets $\nu_{-}=0$.

Consequently is straightforward to verify that $(1,0, \ldots)$, $(0, \ldots, 0,1)$ are equilibria of (13).

Another equilibrium point of (13) is obtained for $\nu_{n}=\frac{\omega}{\beta_{n}}$, with $\frac{1}{\omega}:=\sum_{i=1}^{N} \frac{1}{\beta_{i}}$. Indeed, for this point one has $\nu_{+}=\nu_{-}=$ 0.5 and consequently, the right hand side of (13) becomes $-\frac{\omega}{2}+\frac{\omega}{2}$ or $-\omega+\frac{\omega}{2}+\frac{\omega}{2}$ which are all 0 .

Next, we show that no other equilibrium exists. For this, we suppose by contradiction that there exists some $0<\nu_{+}<1$, $\nu_{+} \neq 0.5$ such that $\nu_{+}=f\left(\nu_{+}\right)$. If such a $\nu_{+}$exists, then that corresponds to another equilibrium point. However, notice that

$$
\frac{\nu_{+}}{1-\nu_{+}}=\frac{\sum_{i=N / 2+1}^{N} \frac{\beta_{1}}{\beta_{i}}\left(\frac{\nu_{+}}{1-\nu_{+}}\right)^{i-1}}{\sum_{i=1}^{N / 2} \frac{\beta_{1}}{\beta_{i}}\left(\frac{\nu_{+}}{1-\nu_{+}}\right)^{i-1}}
$$

replacing $\frac{\nu_{+}}{1-\nu_{+}}$by $\xi$ and accounting for $\beta_{n}=\beta_{N-n+1}$ (symmetry), we must have

$$
\sum_{i=1}^{N / 2} \frac{\beta_{1}}{\beta_{n}}(\xi-1) \xi^{i-1}=0
$$

for an equilibrium point. However, $\xi=1$ corresponds to $\nu_{+}=0.5$. As $\xi>0$, the above equation is never satisfied unless $\beta_{1}=0$ (which also means $\beta_{N}=0$ ). Hence, by contradiction, we prove that the only three equilibrium points are those described.
In order to study the stability of these equilibrium points, we look at the Jacobian and its eigenvalues. If we denote the Jacobian elements by $J_{i, j}$, where $J_{i, j}=\frac{\partial \dot{\nu}_{i}}{\partial \nu_{j}}$, then for all $1<i \leq \frac{N}{2}$, and for all $\frac{N}{2}<j<N$, we have:

$$
\begin{array}{lr}
J_{1,1}=\beta_{2} \nu_{2}-\beta_{1} \nu_{+}, & J_{i, i}=\beta_{i+1} \nu_{i+1}-\beta_{i}, \\
J_{j, j}=\beta_{j-1} \nu_{j-1}-\beta_{j}, & J_{N, N}=\beta_{N-1} \nu_{N-1}-\beta_{N} \nu_{-}
\end{array}
$$

We also have $\forall 2<i \leq N / 2, \quad J_{1, i}=\beta_{2} \nu_{2}$, and

$$
\begin{gathered}
\forall N / 2<i \leq N-2, \quad J_{1, i}=-\beta_{1} \nu_{1}, \\
\forall 2<i \leq N / 2, \quad J_{N, i}=-\beta_{N} \nu_{N}, \\
\forall N / 2<i \leq N-2, \quad J_{N, i}=\beta_{N-1} \nu_{N-1} .
\end{gathered}
$$

For all $i, j \in\{2,3, \ldots, N-1\}$ such that $|i-j|>1$ we have $J_{i, j}=\beta_{i+1} \nu_{i+1}$ when $j \leq N / 2$ and $J_{i, j}=\beta_{i-1} \nu_{i-1}$ when $j>N / 2$. Next, we have $\bar{J}_{1,2}=\beta_{2}\left(\nu_{2}+\nu_{-}\right)$and $J_{N-1, N}=$ $\beta_{N-1}\left(\nu_{N-1}+\nu_{+}\right)$. For all $i, j \in\{2,3, \ldots, N-1\}$ such that $|i-j|=1$ we have

$$
J_{i, i+1}=\beta_{k} \nu_{k}+\beta_{i+1} \nu_{-}
$$

where $k=i+1$ if $i+1 \leq N / 2$ and $k=i-1$ otherwise; and

$$
J_{i, i-1}=\beta_{k} \nu_{k}+\beta_{i-1} \nu_{+}
$$

where $k=i+1$ if $i-1 \leq N / 2$ and $k=i-1$ otherwise.

At the absorbing state $(1,0, \ldots, 0)$, we have the Jacobian evaluated to have diagonal elements to be $0,-\beta_{2}, \beta_{3}$ etc. Additionally, the Jacobian becomes an upper triangular matrix as $\nu_{n}=0$ for all $n>N / 2$. Therefore, the eigenvalues are the elements of the diagonal, which are non-positive and this corresponds to a stable equilibrium. By symmetry, we have the same result for the other absorbing state.

When $\left(\sum_{m=1}^{N / 2} \nu_{m}\right)=0.5$, we have that the fixed point corresponding to this distribution satisfies $\nu_{n} \beta_{n}=\omega$ for some $K>0$. Thus, the first column of the Jacobian can be written as

$$
\left(K-\frac{\beta_{1}}{2}, \omega+\frac{\beta_{1}}{2}, \omega, \ldots, K,-K\right)^{T}
$$

The columns $j$ for $2 \leq j \leq N / 2$ are of the form

$$
\left(\omega, \ldots, \omega+\frac{\beta_{j}}{2}, \omega-\beta_{j}, \omega+\frac{\beta_{j}}{2}, \ldots, \omega,-\omega\right)^{T}
$$

where $\omega+\frac{\beta_{j}}{2}$ is the diagonal term of the Jacobian. The $\mathrm{j}$-th column where $N / 2<j \leq N-1$ is of the form

$$
\left(-\omega, \omega, \ldots, \omega+\frac{\beta_{j}}{2}, \omega-\beta_{j}, \omega+\frac{\beta_{j}}{2}, \ldots, \omega\right)^{T}
$$

Finally, the $N$-th column is given by

$$
\left(-\omega, \omega, \ldots, \omega, \omega+\frac{\beta_{N}}{2}, \omega-\frac{\beta_{N}}{2}\right)^{T}
$$

The above matrix is such that each column has exactly one element which is $-\omega$ either at the first row (after column 
index is more than $N / 2$ ) or at the last row. If we subtract $\omega(N-2) I$ from this matrix, it's determinant becomes 0 . This can be verified using the properties of determinants that adding a scalar times a row to another row does not change the determinant. We replace the first row with the sum of all rows and this results in the first row becoming all zeroes. This implies that one of the eigenvalues of the Jacobian is $\omega(N-2)$ which is positive. Therefore, the equilibrium at $\nu_{+}=\nu_{-}=0.5$ is unstable.

The previous theorem characterizes the behavior of the agents' opinion in all-to-all networks with identical connections. Basically, this result states that beside the two stable equilibria in which all the agents rich consensus we may have a metastable equilibria in which the opinions are symmetrically displaced with respect to 0.5 .

\section{GENERIC INTERACTION NETWORKS}

A way to model generic interaction networks is to consider that they are the union of a number of clusters (see for instance [7] for a cluster detection algorithm). Basically a cluster $C$ is a group of agents in which the opinion of any agent in $C$ is influenced more by the other agents in $C$, than agents outside $C$. When the interactions between cluster are deterministic and very weak, we can use a two time scale-modeling as in [15] to analyze the overall behavior of the network. In the stochastic framework and knowing only a quantized version of the opinions we propose here a development aiming at characterizing the majority actions in clusters. The notion of cluster can be mathematically formalized as follows.

Definition 3 (Cluster): A subset of agents $C \subset \mathcal{K}$ defines a cluster when, for all $i, j \in C$ and some $\lambda>0.5$ the following inequality holds

$$
a_{i j} \geq \lambda \frac{\tau_{i}}{|C|} .
$$

The maximum $\lambda$ which satisfies this inequality for all $i, j \in C$ is called the cluster coefficient.

One question that can be asked in this context is what are the sufficient conditions for the preservation of actions in a cluster, i.e. regardless of external opinions, agents preserve the majority action inside the cluster $C$ for long time. At the limit, all the agents will have identical opinions corresponding to an absorbing state of the network but, clusters with large enough $\lambda$ may preserve their action in metastable states (long time).

For any given set of agents $C \subset \mathcal{K}$, let us denote that

$$
\nu_{-}^{C}=\frac{\sum_{j \in C} \sum_{n=1}^{N / 2} v_{j, n}}{|C|},
$$

and

$$
\nu_{+}^{C}=\frac{\sum_{j \in C} \sum_{n=N / 2+1}^{N} v_{j, n}}{|C|} .
$$

Those values represent the expected fraction of agents within a set $C$ with action 0 and 1 , respectively. We also denote by $\nu_{n}^{C}$, the average probability of agents in a cluster to have opinion $\theta_{n}$, i.e., $\nu_{n}^{C}=\frac{\sum_{i \in C} v_{i, n}}{|C|}$. Now we can use the definition of a cluster given in (16) to obtain the following proposition.

Proposition 2: The dynamics of the average opinion probabilities in a cluster $C \subset \mathcal{K}$ can be written as:

$$
\begin{aligned}
\frac{\dot{\nu}_{1}^{C}}{\kappa}= & -\beta_{1} \nu_{1}^{C}\left(\lambda \nu_{+}^{C}+(1-\lambda) \delta\right) \\
& +\beta_{2} \nu_{2}^{C}\left(\lambda \nu_{-}^{C}+(1-\lambda)(1-\delta)\right) \\
\frac{\dot{\nu}_{n}^{C}}{\kappa}= & -\beta_{n} \nu_{n}^{C}+\beta_{n+1} \nu_{n+1}^{C}\left(\lambda \nu_{-}^{C}+(1-\lambda)(1-\delta)\right) \\
& +\beta_{n-1} \nu_{n-1}^{C}\left(\lambda \nu_{+}^{C}+(1-\lambda) \delta\right) \\
\frac{\dot{\nu}_{N}^{C}}{\kappa}= & -\beta_{N} \nu_{N}^{C}\left(\lambda \nu_{-}^{C}+(1-\lambda)(1-\delta)\right) \\
& +\beta_{N-1} \nu_{N-1}^{C}\left(\lambda \nu_{+}^{C}+(1-\lambda) \delta\right)
\end{aligned}
$$

where $\kappa=\frac{\sum_{i \in C} \tau_{i}}{|C|}$ and $\delta \in[0,1]$.

Proof: Consider $\frac{r_{i}}{\tau_{i}}$ for any agent $i \in C$. We can give a lower bound on it using (16) as

$$
\begin{aligned}
& \frac{r_{i}}{\tau_{i}} \geq \sum_{j \in C} \frac{a_{i j}}{\tau_{i}} \sum_{n=N / 2+1}^{N} v_{j, n} \\
& \Rightarrow \frac{r_{i}}{\tau_{i}} \geq \frac{\lambda}{|C|} \sum_{j \in C} \sum_{n=N / 2+1}^{N} v_{j, n} \\
& \Rightarrow \frac{r_{i}}{\tau_{i}} \geq \lambda \nu_{+}^{C}
\end{aligned}
$$

Applying the same derivation for $l_{i}$, we get $\frac{l_{i}}{\tau_{i}} \geq \lambda \nu_{-}^{C}$. Since $l_{i}+r_{i}=\tau_{i}$, we always have $\lambda \nu_{+}^{C} \leq \frac{r_{i}}{\tau_{i}} \leq \lambda \nu_{+}^{C}+(1-\lambda)$. Thus, we can always rewrite the dynamics of a single agent in the cluster as

$$
\begin{aligned}
\frac{\dot{v}_{i, 1}}{\tau_{i}}= & -\beta_{1} \nu_{1}\left(\lambda \nu_{+}^{C}+(1-\lambda) \delta_{i}\right) \\
& +\beta_{2} \nu_{2}\left(\lambda \nu_{-}^{C}+(1-\lambda)\left(1-\delta_{i}\right)\right) \\
\frac{\dot{v}_{i, n}}{\tau_{i}}= & -\beta_{n} \nu_{n}+\beta_{n+1} \nu_{n+1}\left(\lambda \nu_{-}^{C}+(1-\lambda)\left(1-\delta_{i}\right)\right) \\
& +\beta_{n-1} \nu_{n-1}\left(\lambda \nu_{+}^{C}+(1-\lambda) \delta_{i}\right) \\
\frac{\dot{v}_{i, N}=}{\tau_{i}}= & -\beta_{N} \nu_{N}\left(\lambda \nu_{-}^{C}+(1-\lambda)\left(1-\delta_{i}\right)\right) \\
& +\beta_{N-1} \nu_{N-1}\left(\lambda \nu_{+}^{C}+(1-\lambda) \delta_{i}\right)
\end{aligned}
$$

where $\delta_{i} \in[0,1]$. By taking the sum of each equation over the cluster and dividing by $|C|$, we get the averages. Each term on the right hand side has a constant factor of the type $\left(l_{i}+r_{i}\right) \beta_{m} \nu_{n} \lambda \nu_{+}^{C}$ and an additional perturbation term of the type $\left(l_{i}+r_{i}\right) \beta_{m} \nu_{n}(1-\lambda) \delta_{i}$. The addition of the first type of terms and division by $|C|$ simply becomes $\kappa \beta_{m} \nu_{n} \lambda \nu_{+}^{C}$, with $m$ being $n-1, n$ or $n+1$. The averaging of the perturbation terms results in a value between 0 and $\kappa \beta_{m} \nu_{n}(1-\lambda)$ as all $\delta_{i}$ are in $[0,1]$. This can be therefore be written as in (17) with a new $\delta \in[0,1]$.

The result above shows that instead of looking at individual opinions of agents inside a cluster, we can provide equation (17) for the dynamics of the expected fraction of agents in a cluster with certain opinions. Using proposition 2 and theorem 1 , we can immediately obtain some interesting properties for a cluster.

Corollary 1: Let $C$ be a cluster with coefficient $\lambda \rightarrow 1$. Then the cluster has two metastable equilibria at $\nu^{C}=(1,0, \ldots, 0)$, 
$\nu^{C}=(0, \ldots, 0,1)$ and one unstable equilibrium corresponding to $\nu_{+}^{C}=0.5$.

This result holds as if $\lambda \rightarrow 1$, (17) simply becomes (13) and therefore the equilibrium points of (13) must correspond to those of (17), but with the fraction of population being the fraction of agents within the cluster and not the whole graph. For example, consider that $\mathcal{K}=C_{1} \cup C_{2}$ with $C_{1} \cap C_{2}=\emptyset$ and $\left|C_{1}\right|,\left|C_{2}\right| \rightarrow \infty$. Additionally, $a_{i j}=1$ for all $i, j \in C_{1}$, and for all $i, j \in C_{2}$. Finally, we have a set $A_{1} \subset C_{1}$ and $A_{2} \subset C_{2}$ such that $a_{i j}=0=a_{j i}=0$ for all $i \in C_{1} \backslash A_{1}$ and $j \in C_{2} \backslash A_{2}$, but $a_{i j}=a_{j i}=1$ for all $i \in A_{1}$ and $j \in A_{2}$. If $\left|A_{1}\right|<\infty$ and $\left|A_{2}\right|<\infty$, then we have that $\lambda_{1} \rightarrow 1, \lambda_{2} \rightarrow 1$ which are the cluster coefficients for $C_{1}$ and $C_{2}$. In this example, the graph is connected and so the only two absorbing states must be when all opinions are $\theta_{1}$ or $\theta_{N}$ as shown in proposition 1. Corollary 1 implies that if there are two clusters, each with its $\lambda$ coefficient close to 1 but not 1 , then each cluster can stay with contradicting actions in a metastable state. Such a state is not an absorbing state for the system, but can be held for an arbitrarily long duration.

\section{A. Action preservation}

We have shown that if a cluster has its coefficient $\lambda \rightarrow 1$, then it can preserve its action as if it starts with all agents with opinion $\theta_{1}$ or $\theta_{N}$, this state is a stable state and therefore the local action of the cluster is preserved regardless of the external opinions. However, one problem we want to investigate is if this property hold for other $\lambda<1$. The following proposition provides a necessary condition for a cluster to preserve its action and not collapse to external perturbations.

Proposition 3: A necessary condition for $C$ with coefficient $\lambda$ to preserve its action in a metastable state is if $\exists x \in(0.5,1)$ such that $x=f(\lambda x)$. If no such $x$ exists, then the only equilibrium when the perturbation term $\delta=1$ from (17) is at $\nu_{+}^{C}=1$, and when $\delta=0$, the equilibrium is at $\nu_{+}^{C}=0$.

Proof: The dynamics of the cluster average opinions follow equation (17). We look for equilibrium points for this dynamics under certain values of $\delta$. To find the equilibrium points, we set the left hand side to 0 in (17). For a given $\delta$, by definition of $f(\cdot)$, we obtain that if $\nu_{+}^{C}$ is the fraction of agents with action 1 in the cluster, then it must satisfy

$$
\nu_{+}^{C}=f\left(\lambda \nu_{+}^{C}+(1-\lambda) \delta\right)
$$

Having $\delta=0$ implies that the agents that interact with the agents in the cluster from outside the cluster have all action 0. If $\nu_{+}^{C}>0$ is an equilibrium point even with $\delta=0$, this means that regardless of external opinion, the cluster can preserve an action 1 . This is true because $f(\cdot)$ is a monotonic function and therefore $f\left(\lambda \nu_{+}^{C}+(1-\lambda) \delta\right) \geq f\left(\lambda \nu_{+}^{C}\right)$ for all $\delta \geq 0$.

From the properties of $f(\cdot)$ we know that it is monotonic and takes $f(x)=x$ only when $x=0, .5,1$. Additionally, as $f(x)<x$ when $x \rightarrow 0$, and as $f(\cdot)$ is continuous, $f(\lambda x)<x$ for all $x<0.5$ except at $x=0$. However, $x=0$ corresponds to the state $\nu_{+}=0$ which means that this equilibrium is not preserved. If an $x>0.5$ exists such that $x=f(\lambda x)$, then regardless of the actions outside $C$, i.e. $\delta$, we will have $\nu_{+}^{C} \geq x$ as a possible equilibrium.

By studying the opposite case with the majority action inside the cluster being 0 and external opinion 1 , we get $1-x=f(\lambda(1-x))$ with $1-x>0.5$, which means that the same condition holds for preserving the action 0 as well, to get $\nu_{-}^{C} \geq x$.

\section{B. Propagation of actions}

In the previous subsection, we have seen that a cluster $C$ can preserve its majority action regardless of external opinion if it has a sufficiently large $\lambda$. If there are agents outside $C$ with some connections to agents in $C$, then this action can be propagated. Let $\tau_{i}^{C}=\sum_{j \in C} a_{i j}$ denote the total trust of agent $i$ in the cluster $C$. Let the cluster $C$ be such that it has $\lambda$ large enough so that $\bar{\nu}_{+}^{C}>0.5$ exists where $\bar{\nu}_{+}^{C}=f\left(\lambda \bar{\nu}_{+}^{C}\right)$.

Proposition 4: If the cluster $C$ is preserving an action 1 with at least a fraction $\bar{\nu}_{+}^{C}$ of the population in $C$ having action 1 , then the probability of any agent $i \in \mathcal{K} \backslash C$ to chose action 1 at equilibrium is lower bounded as follows.

$$
\operatorname{Pr}\left(Q_{i}=1\right) \geq f\left(\bar{\nu}_{+}^{C} \frac{\tau_{i}^{C}}{\tau_{i}}\right)
$$

Proof: We know that at equilibrium, we must have:

$$
\sum_{n=N / 2+1}^{N} v_{i, n}=f\left(\frac{r_{i}}{\tau_{i}}\right)
$$

by definition of $f(\cdot)$, (7) and (6). However, we can lower bound $R_{i}$ as

$$
r_{i} \geq \sum_{j \in C} a_{i j} \sum_{n=N / 2+1}^{N} v_{j, n}
$$

Since the cluster $C$ has an action 1 by at least $\bar{\nu}_{+}^{C}$ of its population, we have

$$
\sum_{n=N / 2+1}^{N} v_{j, n} \geq f\left(\lambda \bar{\nu}_{+}^{C}\right)
$$

for any $j \in C$. As $f\left(\lambda \bar{\nu}_{+}^{C}\right)=\bar{\nu}_{+}^{C}$, we have

$$
r_{i} \geq \bar{\nu}_{+}^{C} \sum_{j \in C} a_{i j}
$$

Therefore, $r_{i} \geq \bar{\nu}_{+}^{C} \tau_{i}^{C}$ and so we have

$$
\sum_{n=N / 2+1}^{N} v_{i, n} \geq f\left(\bar{\nu}_{+}^{C} \frac{\tau_{i}^{C}}{\tau_{i}}\right)
$$

\section{NUMERICAL RESULTS}

For all simulations studied, unless otherwise mentioned, we take $\Theta=\{0.2,0.4,0.6,0.8\}, \beta_{1}=\beta_{4}=0.01$ per unit of time and $\beta_{2}=\beta_{3}=0.02$ per unit of time. 


\section{A. General graph}

First, we perform some simulations to validate our NIMFA model for the opinion dynamics. For this purpose, we construct a graph with $K=120$ partitioned into two sets $B_{1}=$ $\{1, \ldots, 40\}$ and $B_{2}=\{41, \ldots, 120\}$. We take $a_{i j}=1$ if a connection exists and 0 otherwise. Connections between agents belonging to the same set $\left(B_{1}\right.$ or $\left.B_{2}\right)$ are established randomly with a probability of 0.3 and connections from $B_{1}$ to $B_{2}$ and vice-versa are established with probability of 0.02 .

For one such randomly generated graph $(\mathcal{K}, A)$, we study the opinion dynamics both by simulating a continuous time Markov chain and by looking at the equilibrium points generated by the recursive search algorithm described in Section III. We always start with the initial state given by

$$
X_{i}(0)=0.2 \text { for all } i \in B_{1}, X_{i}(0)=0.8 \text { for all } i \in B_{2} .
$$

Setting this as the initial condition, the algorithm 1 gives us the population fraction of agents at equilibrium with action 1, i.e., $\nu_{+}$to be 0.693 . To validate this, we run several simulations of the continuous time Markov process with the same initial state and graph structure, and plot the resulting $\sum_{i=1}^{K} Q_{i}(t)$. This plot is shown in Figure 1. We observe that on average over time, the population with action 1 in all simulations are close to the value obtained from the NIMFA model.



Fig. 1: Simulation of $\sum_{i=1}^{K} Q_{i}(t)$ compared to the NIMFA metastable state. The NIMFA works remarkably well even for $K=120$.

Next we focus on the approximation done by NIMFA for individual agent opinions. Table II shows the estimated probability of an agent choosing action 1 for some selected agents. This estimate is computed in each simulation by averaging the action taken over a large time horizon during which the system is in the metastable state. We also observed that the system collapsed to an absorbing state 16 times when we did 1000 simulations. Therefore, we estimate $\operatorname{Pr}\left(Q_{i}=1\right)$ by averaging over the 984 simulations for which the system stayed in the metastable state.

Notice that, as a result of the starting conditions and the graph structure, most agents in $B_{1}$ have a high probability of choosing action 0 at the metastable state (equilibrium of the NIMFA), while most agents in $B_{2}$ chose action 1 . Some agents like 9 and 10 have trust in some agents of $B_{1}$ as well as

\begin{tabular}{|c|c|ccc|c|}
\hline Agent $i$ & \multicolumn{5}{|c|}{ Equilibrium $\operatorname{Pr}\left(Q_{i}=1\right)$ from } \\
& NIMFA & \multicolumn{3}{|c|}{ Simulation $1,2,3$} & Simulation average \\
\hline 1 & 0.003 & 0 & 0 & 0.006 & 0.002 \\
\hline 9 & 0.288 & 0.267 & 0.2 & 0.39 & 0.296 \\
\hline 10 & 0.382 & 0.369 & 0.43 & 0.41 & 0.391 \\
\hline 50 & 0.999 & 0.995 & 0.997 & 0.999 & 0.998 \\
\hline 70 & 1 & 1 & 1 & 1 & 1 \\
\hline 102 & 0.997 & 0.998 & 0.995 & 0.999 & 0.997 \\
\hline
\end{tabular}

TABLE II: $\operatorname{Pr}\left(Q_{i}=1\right)$ at equilibrium for some selected agents. $\operatorname{Pr}\left(Q_{i}=1\right)$ values mentioned in simulation realization 1,2 and 3 are calculated by time averaging over $t=1000$ to $t=3000$ hours and the simulation average is taken over 984 realizations.

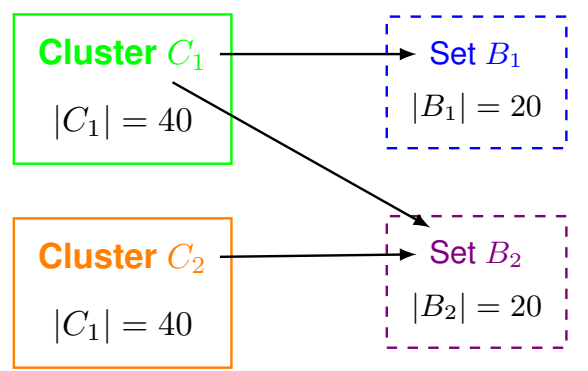

Fig. 2: Structure of the graph. Any two agents in $\mathcal{K}$ may be connected with a 0.05 probability. All agents within a cluster are connected, and the arrows indicate directed connections.

$B_{2}$. Consequently, these agents constantly shift their opinions resulting in a more random behavior for their actions.

\section{B. Graph with clusters}

For the next set of simulations, we take another graph structure, but with the same $\mathcal{K}$ as indicated in Figure 2. We first randomly make links between any $i, j \in \mathcal{K}$ with a probability of 0.05 . When such a link exists, $a_{i j}=1$ and $a_{i j}=0$ otherwise. Then we construct a cluster $C_{1}$, with the agents $i=1,2, \ldots, 40$, and $C_{2}$ with agents $i=81,82, \ldots, 120$. We also label agents $40<i \leq 60$ as set $B_{1}$ and $60<j \leq 80$ as set $B_{2}$. To provide the relevant cluster structure, we made the edge weights $a_{i j}=1$ for all

- $i, j \in C_{1}$ or $i, j \in C_{2}$, making $C_{1}$ and $C_{2}$ clusters with coefficients $\lambda_{1}=0.833$ and $\lambda_{2}=0.816$ (for the particular random graph generated for this simulation).

- $40<i \leq 60$ and $1 \leq j \leq 20$, making agents in $B_{1}$ trust $C_{1}$ with $\frac{\tau_{i}^{C_{1}}}{\tau_{i}} \geq 0.714$ for all $40<i \leq 60$.

- $60<i \leq 80$ and $1 \leq j \leq 20$ or $80<j \leq 120$, making agents in $B_{2}$ trust both $C_{1}$ and $C_{2}$, with $\frac{\tau_{i}^{C_{1}}}{\tau_{i}}, \frac{\tau_{i}^{C_{2}}}{\tau_{i}} \geq$ 0.444 for all $60<i \leq 80$.

We find that the largest $x$ satisfying $x=f\left(\lambda_{1} x\right)$ is 0.95 and that satisfying $x=f\left(\lambda_{2} x\right)$ is 0.94 . Therefore, if all agents in $C_{1}$ start with opinion 0.2 and all agents in cluster 2 start with opinion 0.8 , we predict from proposition 3 that $\nu_{-}^{C_{1}} \geq 0.95$ and $\nu_{+}^{C_{2}} \geq 0.94$ in the metastable state. Additionally, applying proposition 4 yields $\nu_{-}^{B_{1}} \geq f(0.95 \times 0.714)=0.85, \nu_{-}^{B_{2}}, \geq$ $f(0.95 \times 0.444)=0.324$ and $\nu_{+}^{B_{2}} \geq f(0.94 \times 0.444)=0.315$. 


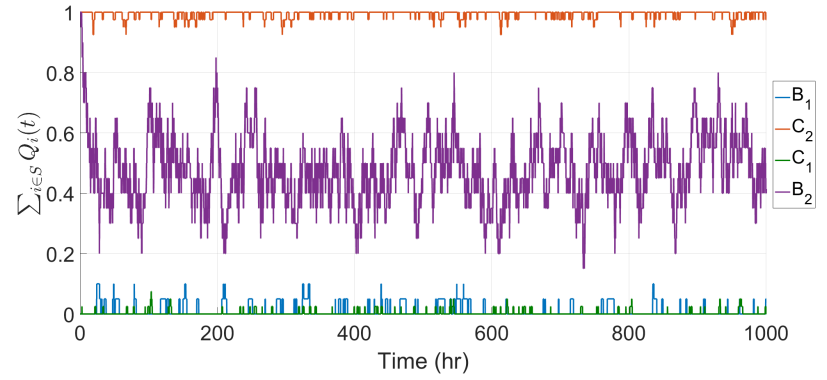

Fig. 3: Simulation of $\sum_{i \in S} Q_{i}(t)$ for $S=C_{1}, C_{2}, B_{1}, B_{2}$. We see that $C_{1}$ and $C_{2}$ preserve their initial actions as given by proposition 3 . We also see that as $B_{1}$ follows only $C_{1}$, it's action is close to $C_{1}$. As $B_{2}$ follows both $C_{1}$ and $C_{2}$ who have contradicting actions, it has a very mixed opinion which keeps changing randomly in time.

Simulations of the continuous time Markov chain show that our theoretical results are valid even when the cluster size is 40 . Figure 3 plots the population fraction of agents with action 1 within a certain set for one simulation. We look for this value in the clusters $C_{1}$ and $C_{2}$ as well as the sets $B_{1}$ and $B_{2} . C_{1}$ and $C_{2}$ are seen to preserve their actions which are opposite to each other. Since $B_{1}$ has a significant trust in $C_{1}$ alone, the opinion of $C_{1}$ is propagated to $B_{1}$. However, as $B_{2}$ trusts both $C_{1}$ and $C_{2}$, its opinion is influenced by the two contradicting actions resulting in having some agents with action 1 and the rest with action 0 . We repeat this for several simulations with the same graph structure and initial opinions to validate our results. Table III compares the predictions based on propositions 3 and 4 with the values obtained in three of our simulations.

\begin{tabular}{|c|c|c|c|c|}
\hline \multirow[t]{2}{*}{ Set $S$} & \multicolumn{4}{|c|}{$\nu_{+}^{S}=E\left[\sum_{i \in S} Q_{i}\right]$ from } \\
\hline & Propositions & & lation & 2,3 \\
\hline$C_{1}$ & $\leq 0.05$ & 0.002 & 0.004 & 0.002 \\
\hline$C_{2}$ & $\geq 0.94$ & 0.994 & 0.995 & 0.995 \\
\hline$B_{1}$ & $\leq 0.15$ & 0.008 & 0.006 & 0.005 \\
\hline$B_{2}$ & $\geq 0.315, \leq 0.685$ & 0.476 & 0.471 & 0.483 \\
\hline
\end{tabular}

TABLE III: $\nu_{+}^{S}$ at equilibrium for the indicated set $S$. Simulation values are calculated by time averaging over $t=100$ to $t=1000$ hours.

\section{CONCLUSION}

In this paper, we have proposed a stochastic multi-agent opinion dynamics model with binary actions. Agents interact through a network and individual opinions are influenced by neighbors action. Our analysis based on a Markov model of opinion dynamics show a consensus like limiting behavior for a finite number of agents. Whereas, when this number becomes large enough, the stochastic system can enter into a quasistationary regime in which partial agreements are reached. This type of phenomenon has been observed in all-to-all and cluster type topologies.

Currently, we have studied the dynamics of opinion without any external control of the network. In the future, we will extend this by accounting for an external entity who has a preferred action (a company selling a product for example) and tries to control the actions of the users in a social network by controlling the opinion of a certain subset of agents. This can be interpreted as a company advertising for its product in order for the other agents in the social network to choose its product over that of a rival company.

\section{REFERENCES}

[1] C. V. Kerckhove, S. Martin, P. Gend, P. J. Rentfrow, J. M. Hendrickx, and V. D. Blondel, "Modelling influence and opinion evolution in online collective behaviour," PLOS ONE, vol. 11, no. 6, pp. 1-25, 2016.

[2] E. Ising, "Contribution to the theory of ferromagnetism," Ph.D. dissertation, University of Hamburg, 1924.

[3] M. H. DeGroot, "Reaching a consensus," Journal of the American Statistical Association, vol. 69, no. 345, pp. 118-121, 1974.

[4] K. Sznajd-Weron and J. Sznjad, "Opinion evolution in closed community,' International Journal of Modern Physics C, vol. 11, no. 6, pp. 1157-1165, 2000.

[5] G. Deffuant, D. Neau, F. Amblard, and G. Weisbuch, "Mixing beliefs among interacting agents," Advances in Complex Systems, vol. 3, pp. 87-98, 2000.

[6] R. Hegselmann and U. Krause, "Opinion dynamics and bounded confidence models, analysis, and simulation," Journal of Artificial Societies and Social Simulation, vol. 5, no. 3, 2002.

[7] I.-C. Morărescu and A. Girard, "Opinion dynamics with decaying confidence: Application to community detection in graphs," IEEE Transactions on Automatic Controlon Automatic Control, vol. 56, no. 8, pp. 1862 - 1873, 2011.

[8] A. Martins, "Continuous opinions and discrete actions in opinion dynamics problems," International Journal of Modern Physics C, vol. 19, no. 4, pp. 617-625, 2008.

[9] N. R. Chowdhury, I.-C. Morărescu, S. Martin, and S. Srikant, "Continuous opinions and discrete actions in social networks: a multi-agent system approach," in Proceedings 55th IEEE Conference on Decision and Control, 2016.

[10] B. S, R. Lima, and T. Araújo, "Agent based models and opinion dynamics as markov chains," arXiv:1108.1716v2, 2011.

[11] J. Lorenz, "Consensus strikes back in the hegselmann-krause model of continuous opinion dynamics under bounded confidence," Journal of Artificial Societies and Social Simulation, vol. 9, no. 1, p. 8, 2006.

[12] P. Van Mieghem, J. Omic, and R. Kooij, "Virus spread in networks," IEEE/ACM Transactions on Networking, vol. 17, no. 1, pp. 1-14, 2009.

[13] S. Trajanovski, Y. Hayel, E. Altman, H. Wang, and P. Van Mieghem, "Decentralized protection strategies against sis epidemics in networks," IEEE Transactions on Control of Network Systems, vol. 2, no. 4, pp. 406-419, 2015.

[14] W. Huisinga, S. Meyn, C. Schutt, H. Wang, and P. Van Mieghem, "Phase transitions and metastability in markovian and molecular systems," The Annals of Applied Probability, vol. 14, no. 1, pp. 419-458, 2004.

[15] S. Martin, I. C. Morărescu, and D. Nešić, "Time scale modeling for consensus in sparse directed networks with time-varying topologies," in Proceedings 55th IEEE Conference on Decision and Control, 2016, pp. $7-12$. 